\title{
Zeaxanthin in Soybean oil: impact of oxidative stability, degradation pattern and product analysis
}

Yunping Yao ${ }^{1}$, Di Zhang ${ }^{1}$, Ruiting $\mathrm{Li}^{1}$, Hang Zhou ${ }^{1}$, Wentao $\mathrm{Liu}^{1}$, Changmo $\mathrm{Li}^{1 *}$ and Shuo Wang ${ }^{2}$

${ }^{1}$ State Key Laboratory of Food Nutrition and Safety, Key Laboratory of Food Nutrition and Safety, Ministry of Education, College of Food Science and Engineering, Tianjin University of Science and Technology, Tianjin 300457, China

${ }^{2}$ Tianjin Key Laboratory of Food Science and Health, School of Medicine, Nankai University, Tianjin, 300071, China

${ }^{*}$ Corresponding Author: Changmo Li

Phone: +86-22-60912486

E-mail: licm@tust.edu.cn 


\section{Summary of Figures and tables in Supporting Information:}

Table S1. Pearson correlation and regression models coefficients relating the stability indexes, $Y=$ peroxide value $(\mathrm{mmol} / \mathrm{kg})$, to the zeaxanthin content, $\mathrm{X}(\mu \mathrm{g} / \mathrm{g})$, in soybean oil.

Table S2. Reaction rate constant $(k)$ and correlation of coefficient $\left(\mathrm{R}^{2}\right)$ of three kinetic models of all-E-zeaxanthin in soybean oil at isothermal condition $\left(110^{\circ} \mathrm{C}\right)$.

Table S3. Zeaxanthin-thermodegraded compounds that tentatively identified in soybean oil.

Figure S1. Possible routes for the formation of 6-methyl-3,5-heptadien-2-one, 3-hydroxy-5,6-epoxy-7,8-dihydro- $\beta$-ionone, 3 -hydroxy- $\beta$-ionone and 3-hydroxy- $\beta$-cyclocitral, from the Zeaxanthin. 
Table S1.

\begin{tabular}{cccccc}
\hline Sample & $\mathbf{1 0}(\boldsymbol{\mu g} / \mathbf{g})$ & $\mathbf{5 0}(\boldsymbol{\mu g} / \mathbf{g})$ & $\mathbf{1 0 0}(\boldsymbol{\mu g} / \mathbf{g})$ & $\mathbf{2 0 0}(\boldsymbol{\mu g} / \mathbf{g})$ & $\mathbf{4 0 0}(\boldsymbol{\mu g} / \mathbf{g})$ \\
\hline $\begin{array}{c}\text { Pearson } \\
\text { correlation }\end{array}$ & -0.954 & -0.981 & -0.988 & -0.960 & -0.995 \\
coefficient & & & & \\
p-value & 0.003 & 0.012 & 0.024 & 0.005 & 0.028 \\
Regression model & $\mathrm{Y}=-4.3886^{*} \mathrm{X}$ & $\mathrm{Y}=-0.9936^{*} \mathrm{X}+$ & $\mathrm{Y}=-0.5726 * \mathrm{X}+$ & $\mathrm{Y}=-0.2368 * \mathrm{X}+$ & $\mathrm{Y}=-0.1137 * \mathrm{X}+$ \\
$\mathbf{R}^{2}$ & +47.315 & 43.217 & 56.95 & 50.543 & 48.923 \\
\hline
\end{tabular}


Table S2.

\begin{tabular}{ccccccc}
\hline & \multicolumn{3}{c}{ Zero-order reaction $^{a}$} & \multicolumn{2}{c}{ The 1st-order reaction $^{b}$} & \multicolumn{2}{c}{ The 2nd-order reaction $^{c}$} \\
\cline { 2 - 7 } Zeaxanthin & \multicolumn{7}{c}{$\mathrm{k} /\left(\mathrm{h}^{-1}\right)\left(\mathrm{R}^{2}\right)$} \\
\hline & $\mathrm{k}$ & $\mathrm{R}^{2}$ & $\mathrm{k}$ & $\mathrm{R}^{2}$ & $\mathrm{k}$ & $\mathrm{R}^{2}$ \\
$10 \mu \mathrm{g} / \mathrm{g}$ & 0.7033 & 0.9275 & 0.1460 & 0.9383 & 0.0372 & 0.8273 \\
$50 \mu \mathrm{g} / \mathrm{g}$ & 3.1206 & 0.9319 & 0.1404 & 0.9482 & 0.0077 & 0.8633 \\
$100 \mu \mathrm{g} / \mathrm{g}$ & 6.7028 & 0.9453 & 0.1325 & 0.9609 & 0.0031 & 0.8686 \\
$200 \mu \mathrm{g} / \mathrm{g}$ & 13.569 & 0.9521 & 0.1451 & 0.9609 & 0.0019 & 0.8603 \\
$400 \mu \mathrm{g} / \mathrm{g}$ & 27.9400 & 0.9805 & 0.1470 & 0.9814 & 0.0009 & 0.8910 \\
\hline
\end{tabular}

a $\quad C_{A}-C_{A, 0}=-k \mathrm{t}$

$b \quad \ln \left(C_{A} / C_{A, 0}\right)=-k \mathrm{t}$

c $\frac{1}{C_{A}-C_{A, 0}}=k \mathrm{t}$

where $C_{A}$ was the zeaxanthin concentration at time $\mathrm{t}$ and $C_{A, 0}$ was the initial zeaxanthin concentration, and the reaction rate constants $\mathrm{k}$ were based on linear regression. 
Table S3.

\begin{tabular}{clc} 
peak & compounds & relative percentage (\%) \\
\hline 1 & 6-methyl- 3,5-heptadien-2-one & 1.77 \\
2 & 3-hydroxy-5,6-epoxy-7,8-dihydro- $\beta$-ionone & 0.49 \\
3 & 3-hydroxy- $\beta$-ionone & 0.35 \\
4 & 3-hydroxy- $\beta$-cyclocitral & 1.02 \\
\hline
\end{tabular}

${ }^{a}$ Data are expressed as percentage values with respect to the total volatile profile. 
Figure S1.

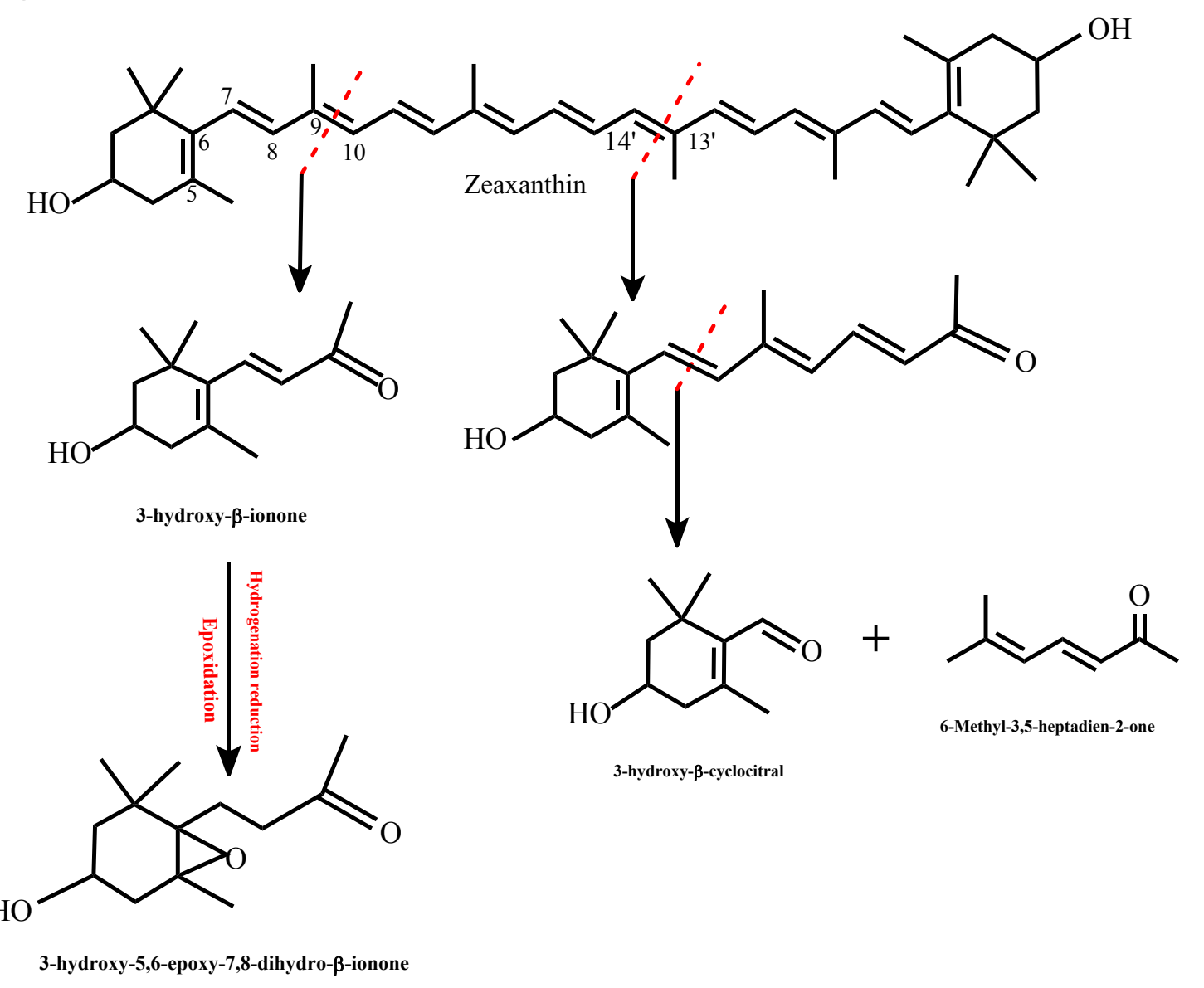

